\title{
DIVERSIDADE E CLASSIFICAÇÃO DA COMUNIDADE ARBÓREA DA FLORESTA OMBRÓFILA MISTA DA FLONA DE SÃO FRANCISCO DE PAULA, RS
}

\author{
CLASSIFICATION AND DIVERSITY OF ARBOREAL COMMUNITY OF THE MOIST \\ OMBROPHYLOUS FOREST IN THE FLONA OF SÃO FRANCISCO DE PAULA FLONA, RS
}

\author{
Sylviane Beck Ribeiro ${ }^{1}$ Solon Jonas Longhi ${ }^{2}$ Doádi Antônio Brena ${ }^{3}$ \\ André R. Terra Nascimento ${ }^{4}$
}

\section{RESUMO}

Este estudo teve como objetivo descrever a diversidade e classificar a comunidade arbórea da Floresta Ombrófila Mista da FLONA de São Francisco de Paula, RS, utilizando dados florísticos e análise hierárquica por meio do programa TWINSPAN (Two-way Indicator Species Analysis). Foram utilizadas dez parcelas de 1 hectare, distribuídas aleatoriamente pela área da FLONA. Em cada conglomerado de 1 hectare, foram levantados todos os indivíduos com DAP $\geq 30 \mathrm{~cm}$. A diversidade de espécies encontrada está dentro dos limites descritos para a Floresta Ombrófila Mista no Sul do Brasil, com estimativas variando de 75 a 110 espécies arbóreas. O método TWINSPAN determinou três grandes grupos florísticos: Grupo 1 - Associação Araucaria que apresentou como espécie indicadora a Araucaria angustifolia (Betol.) Kuntze; Grupo 2 Associação Sebastiania que apresentou como espécies indicadoras Sebastiania commersoniania (Baill.) L. B. Sm. et Downs, Sebastiania brasiliensis Spreng. e Cryptocarya aschersoniana Mez e o Grupo 3 Associação Podocarpus que apresentou como espécie indicadora Podocarpus lambertii Klotzsch ex Endl.

Palavras-chave: Classificação; grupos florísticos; análise hierárquica.

\section{ABSTRACT}

This study aims to describe the diversity and the arboreal community classification of the Ombrophylous Moist Forest located at FLONA in São Francisco de Paula, RS, using floristic and hierarchical analysis data through the TWINSPAN (Two-way Indicator Species Analysis) program. It had been used ten portions of 1 hectare each one and it had been distributed at FLONA's area in a randomnly way. In each 1 hectare conglomerate all individuals with DAP $\geq 30$ had been measured. The encountered species diversity is within the limits that had been described for Ombrophylous Moist Forest in the South of Brazil, with estimates varying from 75 to 110 arboreal species. The TWINSPAN method had determined three great floristic groups: Group 1 - Araucaria association, showing Araucaria angustifolia (Betol.) Kuntze as the indicative species; Group 2 - Sebastiania association, presenting as indicative species Sebastiania commersoniania (Baill.) L. B. Sm. et Downs, Sebastiania brasiliensis Spreng. and Cryptocarya aschersoniana Mez and Group 3 - Podocarpus association, presenting as indicative species Podocarpus lambertii former Klotzsch Endl.

Keywords: Classification; floristic groups; hierarchical analysis.

\section{INTRODUÇÃO}

A concepção de Floresta Ombrófila Mista procede da ocorrência, nessas formações vegetais, da mistura de flora de diferentes origens, definindo padrões fisionômicos típicos em zona climática pluvial. Nessas comunidades, ocorre a coexistência de representantes da flora tropical (Afro-Brasileira) e temperada (Austro-Brasileira), com marcada relevância de elementos Coniferales e Laurales no denominado Planalto Meridional Brasileiro. Esse tipo de vegetação apresenta uma estrutura extremamente variada, ora predominando agrupamentos densos com abundância de Lauráceas e ora agrupamentos pouco desenvolvidos com predomínio de Podocarpus, Drimys e Aqüifoliáceas (Leite e Klein, 1990).

1. Engenheira Florestal, Dra., Professora do Curso de Engenharia Agrícola, Universidade Luterana do Brasil, CEP 68000-000, Santarém (PA). sylviane.ribeiro@ulbranet.com.br

2. Engenheiro Florestal, Dr., Professor do Departamento de Ciências Florestais, Centro de Ciências Rurais, Universidade Federal de Santa Maria, CEP 97105-900, Santa Maria (RS). sjlonghi@smail.ufsm.br

3. Engenheiro Florestal, Dr., Professor Aposentado do Departamento de Ciências Florestais, Centro de Ciências Rurais, Universidade Federal de Santa Maria, CEP 97105-900, Santa Maria (RS).

4. Engenheiro Florestal, Dr., Bolsista PRODOC/CAPES, Programa de Pós-Graduação em Engenharia Florestal, Centro de Ciências Rurais, Universidade Federal de Santa Maria, CEP 97105-900, Santa Maria (RS).

Recebido para publicação em 25/11/2005 e aceito em 17/04/2007. 
Os métodos de classificação da vegetação consistem em agrupar as amostras ou as espécies, segundo suas características. Consiste em dividir o sistema multidimensional em compartimentos ou células, em cada um dos quais se reúnem os pontos que apresentam maior similaridade entre si (Matteucci e Colma, 1982). Para Gauch e Whittaker (1972 e 1981), esse sistema multidimensional é uma matriz primária espéciesparcelas que representa o espaço da espécie, no qual as espécies são os eixos do espaço multidimensional, e as parcelas são os pontos locados por seus escores, para cada espécie.

Sokal e Sneath (1963), Orlóci (1978), Gauch e Whittaker (1981) e Matteucci e Colma (1982) observam que, quando se consideram as características (propriedades que descrevem um indivíduo e que podem assumir um valor) que intervêm na formação dos agrupamentos, as técnicas de classificação podem ser monotéticas e politéticas. As técnicas monotéticas, de acordo com Lambert e Dale (1964) e Lambert e Williams (1962), empregam uma só característica em cada fusão ou subdivisão, enquanto as técnicas politéticas empregam uma função de semelhança ou função objetiva baseada num conjunto de características. O programa TWINSPAN (Two-way Indicator Species Analyis) utiliza um processo multivariado, ou seja, é um método hierárquico, divisivo e politético (Hill, 1979).

Segundo Felfili et al. (2001b), o programa TWINSPAN constrói uma tabela bidimensional pela identificação de espécies preferenciais. As parcelas são classificadas primeiro por dicotimização sucessivas e as espécies pelo mesmo processo. Para obter a dicotimização, as ordenações são divididas pela metade. Esse método faz três ordenações: (i) na ordenação primária, a direção de variação é determinada nos dados; (ii) na segunda ordenação, a dicotomia preliminar é obtida pela divisão da ordenação pela metade e ocorre a identificação de espécies preferenciais (são espécies com as mesmas preferências ecológicas e, dessa forma, sua presença pode ser utilizada para identificar condições ambientais particulares) em um ou outro lado da dicotomia e; (iii) a terceira ordenação é a ordenação por indicador, que é baseada num pequeno número de espécies fortemente preferenciais (Hill, 1979).

Em cada divisão, é calculado um autovalor (eigenvalue) que indica o percentual de variação dos dados da comunidade, explicada no eixo de ordenação. Normalmente, um autovalor em torno de 0,30 é considerado significativo para explicar as variações ocorrentes na natureza, logo, quando a divisão atingir um autovalor menor que 0,30 , esta poderá ser descartada (Kent e Coker, 1992).

Nesse sentido, este trabalho foi realizado com o objetivo de conhecer a diversidade de espécies arbóreas e determinar agrupamentos florísticos distintos, na FLONA de São Francisco de Paula, RS e, assim, fornecer subsídios básicos acerca do comportamento dessas espécies na Floresta Ombrófila Mista, podendo tais informações contribuirem para o seu estudo dentro da Ciência Florestal, mais precisamente nas áreas de Manejo Florestal, Silvicultura e Ecologia.

\section{MATERIAL DE MÉTODO}

\section{Caracterização geral da FLONA}

A Floresta Nacional de São Francisco de Paula (FLONA), local onde foi realizado o estudo (Figura 1), localiza-se a $27 \mathrm{Km}$ da sede do município de São Francisco de Paula, no estado do Rio Grande do Sul, entre as coordenadas geográficas $29^{0} 23^{\prime}$ e $29^{0} 27^{\prime}$ de latitude sul e $50^{\circ} 23^{\prime}$ e $50^{\circ} 25^{\prime}$ de longitude oeste, na serra gaúcha, nordeste do Estado, microrregião dos Campos de Cima da Serra.

A vegetação florestal predominante pertence à Floresta Ombrófila Mista, Mata de Araucária ou Floresta de Pinheiro-brasileiro. Além da Araucaria angustifolia (Bertol.) Kuntze, que imprime um caráter fisionômico nessa vegetação, é comum encontrar outras espécies arbóreas características desse tipo fitogeográfico como o branquilho (Sebastiania commersoniana (Baill.) L. B. Sm. et Downs), cedro (Cedrela fissilis Vell.), pinheiro-bravo (Podocarpus lambertii Klotzsch ex Endl.), dentre outras. A FLONA compreende uma área total em torno de 1.606 ha, sendo que a vegetação nativa cobre cerca de $56 \%$ de sua área total, existindo, ainda, reflorestamentos com Araucaria angustifolia (321 ha), Eucalyptus spp. (34 ha), Pinus elliottii Engelm. (216 ha), Pinus taeda L. (23 ha) e outras espécies vegetais (23 ha) (Teixeira, 2005). 


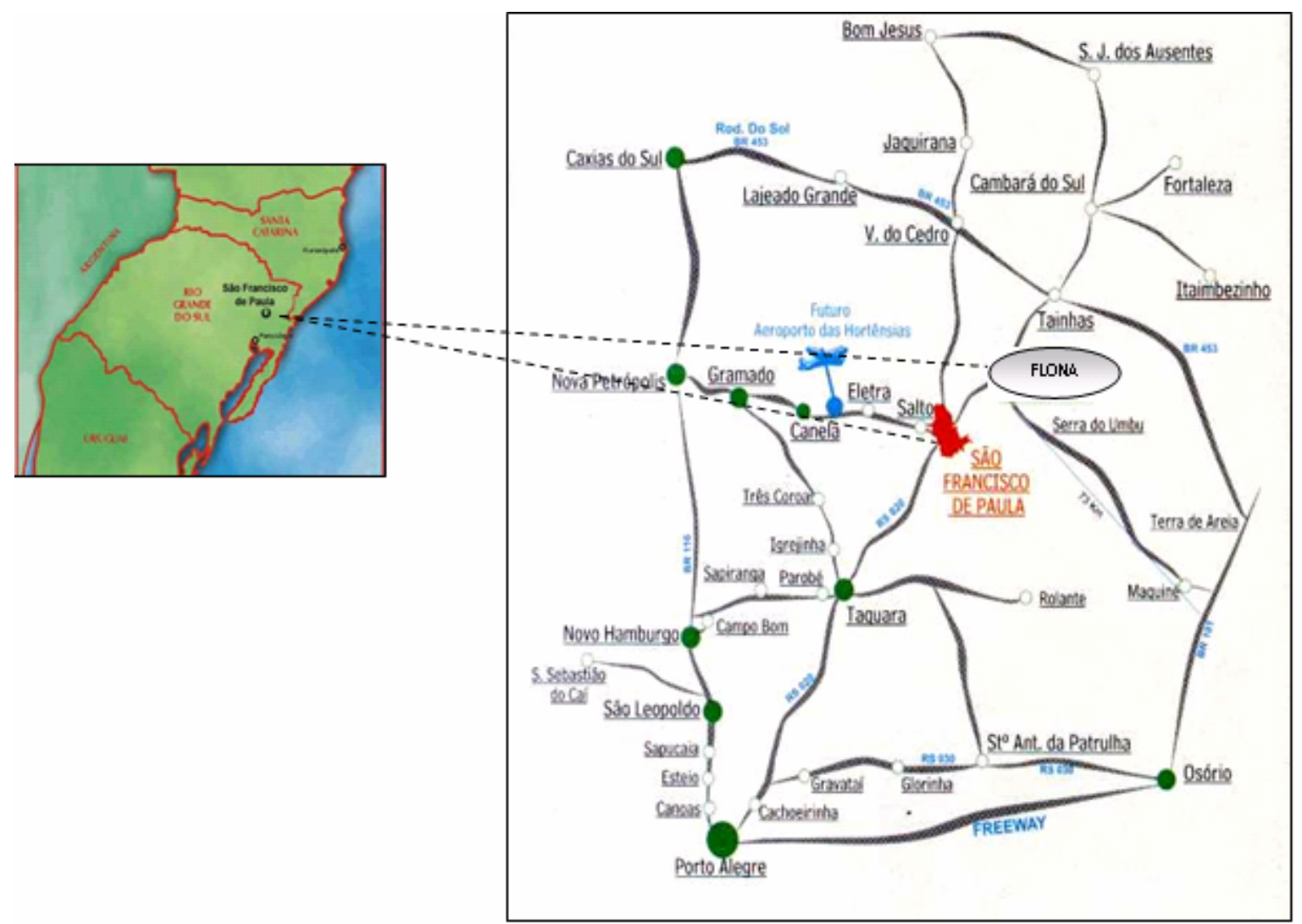

FIGURA 1: Localização da cidade de São Francisco de Paula no estado do Rio Grande do Sul e da FLONA. FIGURE 1: Location of São Francisco de Paula and FLONA in Rio Grande do Sul.

A área referida enquadra-se em clima do tipo "Cfbl", temperado, com invernos rigorosos, registrando-se neves, geadas e nevoeiros em algumas ocasiões e com chuvas todos os meses. A temperatura do mês mais quente é inferior a $22^{\circ} \mathrm{C}$ e do mês mais frio entre $-3^{\circ} \mathrm{C}$ e $18^{\circ} \mathrm{C}$ (Moreno, 1961). A precipitação média anual é de $2.468 \mathrm{~mm}$, e a temperatura média anual é inferior a $18,5^{\circ} \mathrm{C}$.

A região possui relevo ondulado a fortemente ondulado na parte norte, com altitude de $930 \mathrm{~m} \mathrm{e}$ acidentado na parte sul, formando cânions com mais de $100 \mathrm{~m}$ de profundidade. Exemplo disso são as calhas do Arroio Lajeado e da Usina e os peraus do Macaco Branco.

Segundo o Sistema Brasileiro de Classificação de Solos - SBCS, são encontrados na FLONA, os solos CAMBISSOLO HÚMICO Alumínico, CHERNOSSOLO ARGILÚVICO Férrico e NEOSSOLO LITÓLICO Eutrófico (Embrapa, 1999; Streck et al., 2002).

\section{Coleta de dados}

O método de amostragem utilizado foi o de Área Fixa que, segundo Péllico Netto e Brena (1997), é o método que seleciona as árvores a serem amostradas nas unidades amostrais proporcionais à área da unidade e à freqüência dos indivíduos que nela ocorrem. Para a realização do presente trabalho, utilizaram-se dez conglomerados (permanentes) de um hectare. Cada conglomerado é um quadrilátero de $100 \mathrm{~m}$ x $100 \mathrm{~m}$ $\left(10.000 \mathrm{~m}^{2}\right)$ dividido em cem subunidades (parcelas) de $10 \mathrm{~m} \times 10 \mathrm{~m}\left(100 \mathrm{~m}^{2}\right)$.

As parcelas foram instaladas com teodolito, marcando-se os limites externos, as faixas e as subunidades com canos de PVC rígido. Nas subunidades amostrais, levantou-se o estoque de crescimento da floresta, considerando todos os indivíduos com CAP (circunferência à altura do peito) maior ou igual a 30 $\mathrm{cm}$, os quais foram numerados com uma etiqueta de alumínio pregada na base das árvores e registrados: número da árvore, nome da espécie, CAP, altura total e comercial, posição sociológica e coordenadas da árvore. A localização e a instalação das parcelas foi realizada com o auxílio do GPS (Global Positioning System).

As árvores foram registradas pelo seu nome vulgar, quando conhecido, e coletado material botânico 
para posterior identificação no Herbário do Departamento de Ciências Florestais (HDCF) da Universidade Federal de Santa Maria onde foram incorporadas. Eventualmente, algumas espécies foram enviadas ao Departamento de Botânica da Universidade Federal de Minas Gerais, para posterior identificação.

A Circunferência à altura do peito (CAP) das árvores amostradas foram medidas com trena de precisão em milímetros e as alturas com Hipsômetro Digital Vertex. Cada árvore foi classificada também de acordo com a posição que ocupa no estrato em quatro classes: emergente, de dossel, de subdossel e de subbosque.

\section{Classificação dos grupos florísticos (Análise pelo TWINSPAN)}

A classificação dos grupos florísticos dentro das unidades amostrais da Floresta Ombrófila Mista da FLONA foi realizada pelo programa TWINSPAN (Two-way Indicator Species Analysis), o qual verifica a ocorrência de padrões na distribuição de espécies, associados às condições ambientais locais constatadas no campo (Hill, 1979).

$\mathrm{Na}$ análise multivariada dos dados, utilizou-se uma matriz de $998 \times 150$, isto é, contendo na matriz os valores de densidade de 150 espécies incluindo cipós e indivíduos mortos por 998 subunidades (parcelas) amostrais. Duas subunidades foram eliminadas da análise por não conterem nenhum indivíduo com CAP igual ou maior que $30 \mathrm{~cm}$. As parcelas foram classificadas e, posteriormente de forma semelhante, a classificação foi feita para espécies utilizando as amostras como base (Hill et al., 1975; Felfili e Sevilha, 2001; Silva, 2002, Araújo, 2002).

O princípio básico do TWINSPAN é a dicotomia. O programa divide as amostras em grupos por dicotomias sucessivas e, em seguida, faz o mesmo procedimento para as espécies. As etapas da dicotomia podem ser resumidas: (1) achar um primeiro grupo de espécies indicadoras, i.e., que ocorrem juntas nas mesmas amostras, para construir uma ordenação, atribuindo valor 1 a cada uma dessas espécies, e somando os valores para cada amostra; (2) dividir a ordenação num determinado nível, em que seja possível distinguir comunidades de espécies; e (3) dividir as espécies em categorias de acordo com o fato de ser preferencial ou não, dessas comunidades (Valentin, 2000).

Para definição dos agrupamentos, além da análise matemática (multivariada) levaram-se em consideração as características observadas no campo e a possível explicação ecológica para a formação de tais grupos. Conforme Kent e Coker (1992), o número de subdivisões depende do tamanho e da característica do conjunto de dados, considerando que, não necessariamente, todas as divisões são definidas como um grupo, mas aquelas que podem ser verificadas in loco e possuem uma importância ecológica. Conforme os autores para as divisões serem relevantes devem ter um autovalor $\geq 0,30$ (30 \% da variância).

Cada grupo obtido foi caracterizado pela análise florística, considerando a riqueza de espécies e famílias e a diversidade e também por meio de parâmetros biométricos. Para a interpretação da diversidade foi considerado o Índice de Diversidade de Shannon (H'), parâmetro tradicionalmente utilizado em análises fitossociológicas (Daubenmire, 1968; Mueller-Dombois e Ellenberg. 1974; Pielou, 1975). A semelhança entre os grupos foi analisada pelo Índice de Similaridade de Sörensen (Longhi, 1980).

\section{RESULTADOS E DISCUSSÃO}

\section{Classificação por TWINSPAN}

A Análise pelo TWINSPAN definiu três grupos florísticos distintos entre as parcelas da FLONA (Figura 2), os quais diferenciaram-se em relação às suas características ecológicas, que foram determinadas pela densidade das espécies (Crisci e Armengol, 1983). Observa-se que a análise relaciona as espécies indicadoras de cada grupo, que são espécies com preferências ecológicas que podem identificar as condições ambientais das subunidades (Hill et al., 1975).

A primeira grande divisão das subunidades amostrais (parcelas) formou dois grandes grupos. $\mathrm{O}$ autovalor de $0,3659(>0,30)$ mostrou que houve diferenciação entre eles. Essa primeira divisão apontou que existe similaridade florística entre as parcelas relacionadas na esquerda da Figura 2 que, por sua vez, são diferentes das relacionadas na direita, que tendem a apresentar uma maior similaridade. A maioria das espécies não se mostrou exclusivas, porém ocorreram em maior abundância em determinado grupo, de acordo com suas preferências ecológicas. 


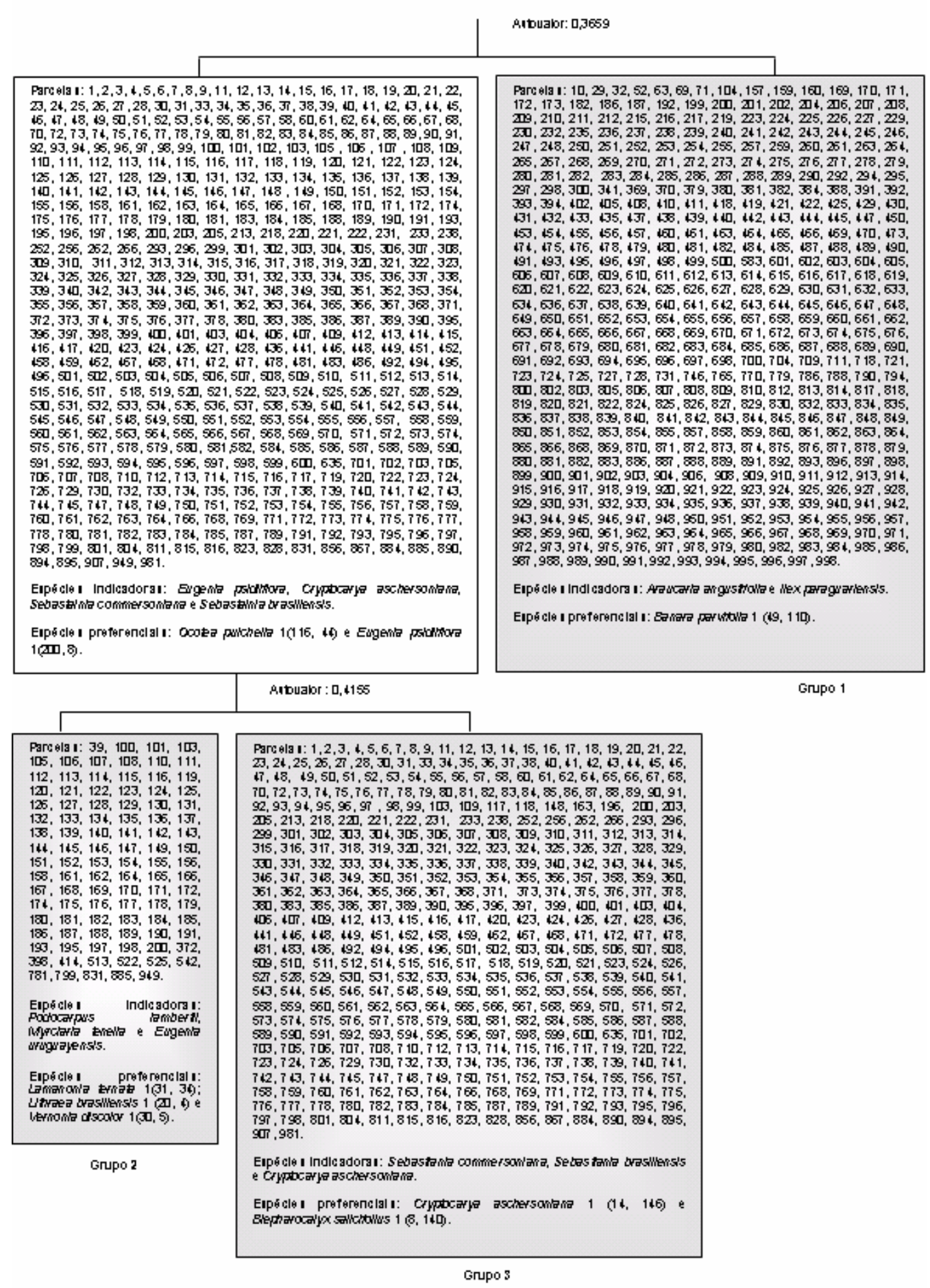

FIGURA 2: Classificação das unidades amostrais avaliadas na FLONA de São Francisco de Paula, RS nos três grupos florísticos.

FIGURE 2: Sample units classification in three floristic groups evaluated at FLONA in São Francisco de Paula, RS.

O primeiro grupo da esquerda apresentou como espécies indicadoras Eugenia psidiiflora O. Berg, Cryptocarya aschersoniana Mez e Sebastiania commersoniana (Baill.) L. B. Sm. et Downs e Sebastiania brasiliensis Spreng.. Esse grupo incluiu a espécie Ocotea pulchella Mart. como espécie preferencial.

No grupo da direita, Araucaria angustifolia e Ilex paraguariensis A. St.-Hil. foram as espécies 
indicadoras, tendo a inclusão de Banara parviflora (A. Gray) Benth. como espécie preferencial. Foi denominado de Grupo I (Associação Araucaria), constituído pela maioria das parcelas amostradas. Pelas exigências ecológicas das espécies constitui o grupo das partes mais altas da área e com uma melhor drenagem do solo.

Na segunda divisão, com um autovalor 0,4155, separou, posteriormente, o grupo da esquerda da primeira divisão em dois grupos.

No grupo menor, à esquerda da segunda divisão, foram observadas como espécies indicadoras Podocarpus lambertii Klotzsch ex. Endl., Myrciaria tenella (DC.) O. Berg e Eugenia uruguayensis Cambess.. As espécies Lamanonia ternata Vell., Lithraea brasiliensis Marchand e Vernonia discolor (Spreng.) Less. foram classificadas como espécies preferenciais. Esse grupo foi denominado de Grupo II (Associação Podocarpus) e constitui o grupo com características intermediárias em relação aos outros dois grupos.

O outro grupo gerado classificou as espécies características de ambientes dos locais mais baixos e com maior umidade do solo. Foi designado de Grupo III (Associação Sebastiania), tendo Sebastiania commersoniania e Sebastiania brasiliensis como espécies indicadoras. Foi também classificada como indicadora a espécie Cryptocarya aschersoniania. As duas espécies do gênero Sebastiania são, segundo Smith et al. (1988), seletivas higrófilas e adaptadas à luz difusa. Segundo Roderjan et al. (2002), essas associações, no domínio da Floresta Ombrófila Mista no sul do Brasil, podem apresentar diferentes graus de desenvolvimento, desde comunidades simplificadas pelo grau de hidromorfia dos solos, onde Sebastiania commersoniania é a espécie característica, até associações mais complexas, em que Araucaria angustifolia tem participação expressiva na fisionomia. Esse grupo teve Blepharocalyx salicifolius (Kunth) O.Berg e Cryptocarya aschersoniana como espécies preferenciais.

As espécies Dicksonia selloviana Hook., Myrceugenia cucullata D. Legrand e Sapium glandulatum (Vell.) Pax., ocorrentes nesse grupo são também segundo Schnem (1978), Legrand e Klein (1970) e Smith et al. (1988) respectivamente adaptadas a solos úmidos.

Os agrupamentos formados geraram novas divisões, porém, pelas observações de campo e pelos objetivos desejados no estudo, considerou-se a diferenciação de três grupos florísticos distintos, que demonstraram claramente as características ecológicas da área.

\section{Diversidade florística do componente arbóreo}

Nos dez conglomerados de 1 hectare, foram amostradas um total de 130 espécies arbóreas com CAP $\geq 30 \mathrm{~cm}$, pertencentes a 79 gêneros e 45 famílias botânicas. Os parâmetros fitossociológicos por cada grupo florístico podem ser observados na Tabela 1, com as maiores estimativas para a riqueza de espécies, área basal e volume por hectare encontrados na formação Araucaria onde essa espécie arbórea apresenta uma maior participação na biomassa desse grupo florístico.

Como a área da amostragem é extensa (10 hectares) foi observado um elevado número de espécies arbóreas para as condições da Floresta Ombrófila Mista, com valores entre 75 e 110 espécies (CAP $\geq 30 \mathrm{~cm})$ entre os três grupos. Por outro lado, as estimativas de diversidade de Shannon (H'), variando de 1,46 a 1,79 foram extremamente baixas e reportam os resultados de Jarenkow e Batista (1987) que mencionam um valor superior $\left(H^{\prime}=2,93\right)$ e colocam esse valor como um dos mais baixos já registrados para florestas naturais com o componente arbóreo multiestratificado.

A similaridade florística entre os grupos foi alta, com todas as comparações acima de 0,5 (Tabela 2), valor este considerado como elevado para comparações entre comunidades vegetais (Kent e Coker, 1992). A maior similaridade foi encontrada entre os grupos 2 e $3(\mathrm{Ss}=0,75)$, os quais apresentam uma menor expressão da espécie Araucaria angustifolia (Bertol.) Kuntze e possuem um menor número de espécies exclusivas de cada grupo (Tabela 1). Os altos índices de similaridade encontrados entre os grupos demonstram que existe um expressivo número de espécies arbóreas comuns entre os grupos, as quais possuem adaptação as situações ecológicas variadas encontradas na área estudada.

TABELA 1: Parâmetros fitossociológicos por cada grupo florístico na FLONA de São Francisco de Paula, RS. Valores entre parênteses representam a porcentagem do total das espécies. 
TABLE 1: Phytosociological parameters for each floristic group at FLONA in São Francisco de Paula, RS. Values in parentheses represent the total species percentage.

\begin{tabular}{l|cccc}
\hline Parâmetros & Associação Araucaria & Associação Podocarpus & Associação Sebastiania \\
\hline Riqueza de espécies & 110,0 & 75,0 & 96,0 \\
N. de Famílias & 39,0 & 29,0 & 38,0 \\
Diversidade (H') & 1,46 & 1,79 & 1,62 \\
Espécies exclusivas & $25,0(22,7 \%)$ & $3,0(4,0 \%)$ & $8,0(8,3 \%)$ \\
Área basal (m ${ }^{2} / \mathrm{h}$ a) & 47,38 & 41,68 & 44,86 \\
N. de Ind./ha & 827,5 & 1031,3 & 856,3 \\
Volume $\left(\mathrm{m}^{3} / \mathrm{ha}\right)$ & 413,6 & 252,3 & 274,8 \\
\hline
\end{tabular}

TABELA 2: Similaridade de Sörensen entre os três grupos florísticos na FLONA de São Francisco de Paula, RS.

TABLE 2: Sörensen similarity between three floristic groups at FLONA in São Francisco de Paula, RS.

\begin{tabular}{c|c|c|c}
\hline \multirow{2}{*}{ Grupos } & \multicolumn{3}{|c}{ Similaridade entre Grupos (Sörensen) } \\
\cline { 2 - 4 } & 1 & 2 & 3 \\
\hline 1 & 1 & 1 & \\
3 & 0,69 & 0,75 & 1 \\
\hline
\end{tabular}

\section{CONCLUSÕES}

Com base nos resultados do presente estudo e para as condições da Floresta Ombrófila Mista da FLONA, pode-se concluir:

A classificação hierárquica gerada pelo TWISNPAN identificou a existência de três grandes grupos

florísticos: I. Associação Araucaria, II. Associação Podocarpus e III. Associação Sebastiania relacionados, em pequena escala, com a densidade destas espécies indicadoras de cada grupo e com as condições ambientais distintas existentes na área.

Entre os três grupos florísticos identificados na FLONA o grupo I (Associação Araucaria) apresentou os maiores valores de riqueza de espécies arbóreas, área basal e volume por hectare. Embora os valores de diversidade encontrados fossem baixos $\left(\mathrm{H}^{\prime}=1,64\right)$, esse grupo apresentou um percentual expressivo de espécies exclusivas $(22,7 \%)$, muito superior aos outros dois grupos encontrados e pode ser considerado o estágio de sucessão avançada da Floresta Ombrófila Mista na região.

\section{REFERÊNCIAS BIBLIOGRAFICAS}

ARAÚJO, M. M. Vegetação e mecanismos de regeneração em fragmento de Floresta Estacional Decidual Ripária, Cachoeira do Sul, RS, Brasil. 2002. 154f. Tese (Doutorado em Engenharia Florestal) - Universidade Federal de Santa Maria, Santa Maria, 2002.

CRISCI, J. V.; ARMENGOL, M. F. L.; Introducion a la teoria y practica de la taxonomia numerica. Washington: Secretaría General de la Organización de los Estados Americanos, Programa Regional de Desarrollo Científico y Tecnológico, 1983. 131p.

DAUBENMIRE, R. Plant communities : a textbook of plant synecology. New York: Harper \& Row, 1968. 300p.

EMBRAPA. Centro Nacional de Pesquisa de Solos. Sistema Brasileiro de Classificação de Solos. Brasília: EMBRAPA-SPI, 1999. 412p.

FELFILI, J. M.; SEVILHA, A. C. Distribuição espacial de parcelas e de espécies em quatro áreas de cerrado sensu stricto na Chapada do Espigão Mestre de São Francisco. In: FELFILI, J. M.; SILVA JR., M. C. da (org.) Biogeografia do bioma Cerrado: estudo fitofisionômico na Chapada do Espigão Mestre do São Francisco. Brasília: UnB, 2001. p.61-74.

FELFILI, J. M.; SILVA JR., M. C. da; REZENDE, A. V.; HARIDASAN, M.; FILGUEIRAS, T. S.; MENDONÇA, R. C. da; WALTER, B. M. T.; NOGUEIRA, P. E. O projeto Biogeografia do Bioma Cerrado: hipótese e padronização da metodologia. In: GARAY, I.; DIAS, B. F. S. (eds.). Conservação da biodiversidade em ecossistemas tropicais. Petrópolis: Vozes, 2001. p.157-173.

GAUCH, H. G.; WHITTAKER, R. H. Comparison of ordination techniques. Ecology, Ithaca, v.53, n.5, p.868-875, 1972. 
GAUCH, H. G.; WHITTAKER, R. H. Hierarchical classification of community data. Journal of Ecology, Oxford, v.69, n.2, p.573-597, 1981.

HILL, M. O. TWINSPAN: a FORTRAN program fo arranging multivariate data in an ordered two way table by classification of individual and attributes. Ithaca : Cornell University, 1979.60p.

HILL, M. O.; BUNCE, R. G. H.; SHAW, M. W. Indicator species analysis, a divisive polythetic method of classification, and its application to a survey of native pinewoods in Scotland. Journal of Ecology, v. 63, n.2, p.597$613,1975$.

JARENKOW, J.A.; BATISTA, L.R.M. 1987. Composição florística e estrutura da Mata com Araucária na Estação Ecológica de Aracuri, Esmeralda, Rio Grande do Sul. Napaea, n.3, p.9-18, 1987.

KENT, M.; COKER, P. Vegetation description analyses. London: Behaven Press, 1992. 363p.

LAMBERT, J. M. ; DALE, M. B. The use of statistics in phytossociology. Adv. Ecol. Res., v.2, p.59-99, 1964.

LAMBERT, J. M.; WILLIAMS, W. T. Multivariate methods in plant ecology. IV-Nodal analysis. Journal of Ecology, Oxford, v.50, n.4-6, p.775-802, 1962.

LEGRAND, C. D.; KLEIN, R. M. Mirtáceas: 3. Myrceugenia. In: REITZ, R. (ed.). Flora Ilustrada Catarinense. Itajaí: Herbário Barbosa Rodrigues, 1970. 453p.

LEITE, P.F.; KLEIN, R.M. Vegetação. In: IBGE. Instituto Brasileiro de Geografia e Estatística. Geografia do Brasil: Região Sul. Rio de Janeiro, 1990. p.113-150.

LONGHI, S. J. A estrutura de uma floresta natural de Araucaria angustifolia (Bert.) O. Ktze., no Sul do Brasil. 1980. 198f. Dissertação (Mestrado em Ciências Florestais) - Universidade Federal do Paraná, Curitiba, 1980.

MATTEUCCI, S. C.; COLMA, A. Metodologia para el estudio de la vegetacion. Washington: Secretaria General de la Organizción de los Estados Americanos - Programa Regional de Desarrolo Científico y Tecnológico, 1982. 169p.

MORENO, J.A. Clima do Rio Grande do Sul. Porto Alegre: Secretaria da Agricultura, 1961. 42p.

ORLÓCI, L. Multivariate analysis in vegetation research. 2.ed. The Hague: DR. W. Junk B. V. Publishers, 1978. 415p.

MUELLER-DOMBOIS, D.; ELLENBERG, H. Aims and methods of vegetation ecology. New York: John Wiley \& Sons, 1974. 547p.

PÉLLICO NETTO, S.; BRENA, D. A. Inventário florestal. Curitiba: Ed. dos Autores, 1997. 245p.

PIELOU, E. C. Ecological diversity. New York : John Wiley, 1975. 165p.

RODERJAN, C. V.; GALVÃO, F.; KUNIYOSHI, Y.S.; HATSCHBACH, G.G. As unidades fitogeográficas do Estado do Paraná. Ciência \& Ambiente, Santa Maria, v.24, p.75-92, 2002.

SCHNEM, A. S. J. Ciateáceas. In: REITZ, R. (ed.). Flora Ilustrada Catarinense. Itajaí: Herbário Barbosa Rodrigues, 1978. 115 p.

SILVA, P. E. N. da. Florística, fitossociologia e nutrição mineral do cerrado sentido restrito no Complexo Xavantina - MT. 2002. Tese (Doutorado em Ecologia) - Universidade de São Paulo, São Paulo, 2002.

SMITH, L. B.; DOWNS, R. J.; KLEIN, R. M. Euforbiáceas. In: Reitz, R. Flora Ilustrada Catarinense. Itajaí: Herbário Barbosa Rodrigues, 1988. p.308-313.

SOKAL, R. R.; SNEATH, P. H. Principles of numerical taxonomy. San Francisco: Freeman, 1963. 450p.

STRECK, E. V. et al. Solos do Rio Grande do Sul. Porto Alegre: EMATER/RS; UFRGS, 2002. 107p.

TEIXEIRA, I. F. Estudo da paisagem da Floresta Nacional de São Francisco de Paula, RS, Brasil. 2005. $212 f$. Tese (Doutorado em Engenharia Florestal) - Universidade Federal de Santa Maria, Santa Maria, 2005.

VALENTIN, J. L. Ecologia numérica uma introdução à análise multivariada de dados ecológicos. Rio de Janeiro : Editora Interciência, 2000.117p. 